\title{
Existence and Non-Existence Result for Singular Quasilinear Elliptic Equations*
}

\author{
Mingzhu Wu ${ }^{1}$, Zuodong Yang ${ }^{1,2}$ \\ ${ }^{1}$ Institute of Mathematics, School of Mathematical Science, Nanjing Normal University, \\ Nanjing, China \\ ${ }^{2}$ College of Zhongbei, Nanjing Normal University, Nanjing, China \\ E-mail: zdyang_jin@263.net \\ Received June 1, 2010; revised August 11, 2010; accepted August 14, 2010
}

\begin{abstract}
We prove the existence of a ground state solution for the qusilinear elliptic equation $-\operatorname{div}\left(|\nabla u|^{p-2} \nabla u\right)$ $=f(x, u)$ in $\Omega \subset \mathbf{R}^{N}$, under suitable conditions on a locally Holder continuous non-linearity $f(x, t)$, the non-linearity may exhibit a singularity as $t \rightarrow 0^{+}$. We also prove the non-existence of radially symmetric solutions to the singular elliptic equation $-\operatorname{div}\left(|\nabla u|^{p-2} \nabla u\right)=d(x)\left[g(u)+r(u)+|\nabla u|^{q}\right], \quad u(x)>0 \quad$ in $\quad \mathbf{R}^{N}$, $u(x) \rightarrow 0 \quad$ as $\quad|x| \rightarrow \infty$, where $d(x)=d(|x|) \in C\left(\mathbf{R}^{N},(0, \infty)\right), \quad N \geq 3, q \geq 0 . g \in C^{1}((0, \infty),(0, \infty)), r \in C_{\text {loc }}^{0, \alpha}([0, \infty)$, $[0, \infty)), 0<\alpha<1$.
\end{abstract}

Keywords: Quasilinear Elliptic Equations, Existence, Non-Existence, Singular

\section{Introduction}

In this paper, we are concerned with the existence of ground state solution or positive solution for the following problem

$$
\left\{\begin{array}{l}
-\operatorname{div}\left(|\nabla u|^{p-2} \nabla u\right)=f(x, u), x \in \Omega \\
u>0, x \in \Omega \\
u(x)=0, x \in \partial \Omega
\end{array}\right.
$$

in which $\Omega \subset \mathbf{R}^{N}, u(x) \rightarrow 0$ when $|x| \rightarrow \infty$. Let $f: \Omega \times[0, \infty) \rightarrow[0, \infty)$ be a locally Holder continuous function which may be singular at $t=0$.

The problem (1) appears in the study of non-Newtonian fluids [1,2] and non-Newtonian filtration [3]. The quantity $p$ is a characteristic of the medium. Media with $p>2$ are called dilatant fluids and those with $p<2$ are called pseudoplastics. If $p=2$, they are Newtonian fluid. When $p \neq 2$, the problem becomes more complicated since certain nice properties inherent to the case $p=2$ seem to be lose or at least difficult to verify. The main differences between $p=2$ and $p \neq 2$ can be found in [4-6].

In recent years the study of ground state solutions for

*Project Supported by the National Natural Science Foundation of China (No.10871060); the Natural Science Foundation of Educational Department of Jiangsu Province (No.08KJB110005) $p=2$ has received a lot of interest and gets numerous existence results (see [7-12]). For $p$-Laplacian equations, in most papers, the focus has been on separable nonlinearities like (2).

$$
\left\{\begin{array}{l}
-\operatorname{div}\left(|\nabla u|^{p-2} \nabla u\right)=b(x) g(u), x \in \mathbf{R}^{N} \\
u>0, \quad x \in R^{N} \\
u(x) \rightarrow 0, \text { as }|x| \rightarrow \infty
\end{array}\right.
$$

We refer readers to the paper [13-19]. In this paper, we consider the situation of $p>1$.

In [20], the author extended results to the problem (1) for $p=2$ where $f$ is not necessarily separable. For $p \geq 2$ we can see [1-6]. Motivated by papers [4-6,20], we extend the results to $p>1$ and get two theorems. But we still have many difficulties to get entire ground state solution of (1).

The second purpose is to give a result for nonexistence of solution. To the best of our knowledge, there has been very less result for nonexistence of solution about singular elliptic equation. We solve an open problem in [13] for $p>1$, for the case when $u$ is a radially symmetric solution.

The paper is organized as follows. In Section 2 we recall some facts and give many lemmas that will be needed in the paper. In Section 3, we give the proof of the main result of the paper. 


\section{Preliminaries}

Firstly we list the following assumptions and results that needed below.

$$
\left\{\begin{array}{l}
-\operatorname{div}\left(|\nabla W|^{p-2} \nabla W\right)=b(x), x \in \mathbf{R}^{N} \\
W>0, x \in R^{N} \\
W(x) \rightarrow 0, \text { as }|x| \rightarrow \infty
\end{array}\right.
$$

$\left[B_{1}\right] b>0$ is a locally Holder continuous function on $\mathbf{R}^{N}$.

$$
\left[B_{2}\right] \int_{0}^{\infty}\left(t^{1-N} \int_{0}^{t} s^{N-1} b^{*}(s) d s\right)^{\frac{1}{p-1}} d t<\infty, \text { where }
$$

$b^{*}(t)=\max \{b(x):|x|=t\}$ for $t>0$.

$\left[B_{3}\right]$ Problem (3) has a solution $W_{b}$ in $\mathbf{R}^{N}$ when $b$ satisfy some condition.

It can be proved that condition $\left[B_{2}\right]$ implies $\left[B_{3}\right]$. This follows from the observation that $v(r) \rightarrow 0$ as $r \rightarrow \infty$ where

$$
v(r)=\int_{r}^{\infty}\left(t^{1-N} \int_{0}^{t} s^{N-1} b^{*}(s) d s\right)^{\frac{1}{p-1}} d t
$$

and satisfies the following equation

$-\operatorname{div}\left(|\nabla v|^{p-2} \nabla v\right)=b^{*}(x)$.

Thus $w(x)=v(|x|)$ is a supersolution of (3). Since $0 \leq w$, and zero is clearly a sub-solution it follows from standard results that (3) admits a solution $W_{b}$ such that $0<W_{b} \leq w$.

The following eigenvalue problem

$$
\left\{\begin{array}{l}
-\operatorname{div}\left(|\nabla \phi|^{p-2} \nabla \phi\right)=\lambda c(x) \phi^{p-1}, x \in \mathbf{R}^{N} \\
\phi \rightarrow 0, \text { as }|x| \rightarrow \infty
\end{array}\right.
$$

where $\Omega \subseteq \mathbf{R}^{N}$ is a bounded smooth domain, and $c \in C^{\alpha}(\bar{\Omega},(0, \infty))$ for some $0<\alpha<1$. The first eigenvalue of the problem (4) will be denoted by $\lambda_{\Omega}>0$. It is easily noted from the variational characterization of eigenvalues that $\lambda_{\Omega_{1}} \geq \lambda_{\Omega_{2}}$ where $\Omega_{1} \subseteq \Omega_{2}$ are smooth bounded domains in $\mathbf{R}^{N}$.

Consider $g:(0, \infty) \rightarrow(0, \infty)$ satisfies the following conditions:

$\left[G_{1}\right] g$ is $C^{1}$.

$\left[G_{2}\right] \limsup _{t \rightarrow \infty} \frac{g(t)}{t}<\frac{1}{\left\|W_{b}\right\|_{\infty}}$, where

$\left\|W_{b}\right\|_{\infty}=\max _{x \in \mathbf{R}^{N}} W_{b}(x)$.

The nonlinearity $f$ in problem (1) is assumed to be a real function that satisfies the following conditions: $\left[F_{1}\right] f(x, t)$ is locally Holder continuous on $\mathbf{R}^{N} \times(0, \infty)$ and continuously differentiable in the variable $t$.

$\left[F_{2}\right] f(x, s) \leq b(x) g(s)$ for all $(x, s) \in \mathbf{R}^{N} \times(0, \infty)$ and some functions $b: \mathbf{R}^{N} \rightarrow(0, \infty)$ and $g:(0, \infty) \rightarrow(0, \infty)$

$\left[F_{3}\right]$ There is a continuous function $a: \mathbf{R}^{N} \rightarrow(0, \infty)$ and some constants $\vartheta>0$ and $\lambda>\lambda_{1}$ such that

$$
f(x, s) \geq \lambda a(x) s^{p-1},(x, s) \in \mathbf{R}^{N} \times(0, \vartheta)
$$

where $\lambda_{1}$ is the first eigenvalue of the problem (4) on the ball $B(0,1)$ of radius one and centered at the origin and where $c(x)=a(x)$.

Recall that the nonlinearity $f(x, t)$ may exhibit singularity as $t \rightarrow 0^{+}$. We will consider the following Dirichlet problem for a given smooth bounded domain $\Omega \subseteq \mathbf{R}^{N}$.

$$
\left\{\begin{array}{l}
-\operatorname{div}\left(|\nabla W|^{p-2} \nabla W\right)=b(x), x \in \Omega \\
W>0, \quad x \in \Omega \\
W(x)=0, \quad x \in \partial \Omega
\end{array}\right.
$$

To establish the main theorem, from reference [2] we give the following lemmas.

Lemma 1. (Weak Comparison Principle) Let $\Omega$ be a bounded domain in $\mathbf{R}^{N}(N \geq 2)$ with smooth boundary $\partial \Omega$ and $\theta:(0, \infty) \rightarrow(0, \infty)$ is continuous and nondecreasing. Let $u_{1}, u_{2} \in W_{l o c}^{1, p}(\Omega) \cap C(\Omega)$ satisfy

$$
\begin{aligned}
& \int_{\Omega}\left|\nabla u_{1}\right|^{p-2} \nabla u_{1} \nabla \psi d x+ \\
& \int_{\Omega} \theta\left(u_{1}\right) \psi d x \leq \int_{\Omega}\left|\nabla u_{2}\right|^{p-2} \nabla u_{2} \nabla \psi d x+\int_{\Omega} \theta\left(u_{2}\right) \psi d x
\end{aligned}
$$

for all non-negative $\psi \in W_{l o c}^{1, p}(\Omega)$. Then the inequality

$\lim _{x \rightarrow \partial \Omega} \sup \left(u_{1}(x)-u_{2}(x)\right) \leq 0$

implies that

$u_{1} \leq u_{2}$ in $\Omega$.

Before Lemma 2, we give the following equation

$$
-\operatorname{div}\left(|\nabla u|^{p-2} \nabla u\right) \pm f(x, u)=0, \quad x \in \mathbf{R}^{N}
$$

Lemma 2. Suppose that $f(x, u)$ is defined on $\mathbf{R}^{N+1}$ and is locally Holder continuous (with exponent $\lambda \in(0,1))$ in $x$. Suppose moreover that there exist functions $v, w \in C_{\text {loc }}^{1+\lambda}\left(\mathbf{R}^{N}\right)$ such that

$$
\left\{\begin{array}{l}
-\operatorname{div}\left(|\nabla v|^{p-2} \nabla v\right) \pm f(x, v) \leq 0 \\
-\operatorname{div}\left(|\nabla w|^{p-2} \nabla w\right) \pm f(x, w) \geq 0
\end{array}\right.
$$

and

$$
v(x) \leq w(x)
$$

and that $f(x, u)$ is locally Lipschitz continuous in $u$ on the set

$\left\{(x, u): x \in \mathbf{R}^{N}, v(x) \leq u \leq w(x)\right\}$

Then Equation (6) possesses an entire solution $u(x)$ satisfying

$$
v(x) \leq u(x) \leq w(x), \quad x \in \mathbf{R}^{N}
$$

Lemma 3. Let $b$ satisfy $\left[B_{1}\right],\left[B_{3}\right]$ and $g$ satisfy both $\left[G_{1}\right],\left[G_{2}\right]$. Then there is $v \in C^{1}\left(\mathbf{R}^{N}\right)$ such 
that

$$
-\operatorname{div}\left(|\nabla v|^{p-2} \nabla v\right) \geq b(x) g^{p-1}(v(x)),
$$$$
v>0, v(x) \rightarrow 0 \text { as }|x| \rightarrow \infty \text {. }
$$

Proof. Since $g$ satisfies $\left[G_{2}\right]$, we define

$$
\hat{g}(t):=\sup \left\{\frac{g(s)}{s}: s>t\right\}, t>0
$$

Note that $\hat{g}(t)$ is non-increasing, positive and $\hat{g}(t) \geq g(t) t^{-1}$. Furthermore, by $\left[G_{2}\right]$ we have $\hat{g}(t)<\left\|W_{b}\right\|_{\infty}^{-1}$ for sufficiently large $t$.

Let

$$
h(t)=\frac{2}{t} \int_{\frac{t}{2}}^{t} \hat{g}(s) d s, t>0
$$

Then $h$ is $C^{1}$, non-increasing and $\hat{g}(t) \leq h(t) \leq \hat{g}\left(\frac{t}{2}\right)$ for all $t \in(0, \infty)$. Since $h$ is nonincreasing, we note that $h(t) \rightarrow \alpha<\left\|W_{b}\right\|_{\infty}^{-1}$ as $t \rightarrow \infty$ for some $\alpha \in[0, \infty)$. Now, let us set

$$
\eta(t):=\int_{0}^{t} \frac{1}{h(s)} d s, t>0
$$

On using $\hat{g}(t)<\left\|W_{b}\right\|_{\infty}^{-1}$ in (8) for sufficiently large $t>0$, we see from (9) that

$$
\eta(\gamma)>\gamma\left\|W_{b}\right\|_{\infty}
$$

for a sufficiently large $\gamma \geq 1$. Let $\psi=\eta^{-1}$ be the inverse function of $\eta$.

By direct calculation, we see that $\psi^{\prime}(t)=h(\psi(t)), \psi(t)>0$, for $t>0$ and $\psi(0)=0$.

Furthermore

$\psi^{\prime \prime}(t)=h(\psi(t)) h^{\prime}(\psi(t)), t>0$.

By condition $\left[B_{3}\right]$, we take a solution $W_{b}$ of (3) with $\Omega=\mathbf{R}^{N}$. Let us now set $v(x):=\psi\left(\gamma W_{b}(x)\right)$ for all $x \in \Omega$. We note from (10) that

$$
v(x)=\psi\left(\gamma W_{b}(x)\right) \leq \psi\left(\gamma\left\|W_{b}\right\|_{\infty}\right)<\gamma
$$

A simple computation shows that $v$ has the desired properties.

Indeed, on recalling $-\operatorname{div}\left(\left|\nabla W_{b}\right|^{p-2} \nabla W_{b}\right)=b$, we see that

$$
\begin{aligned}
- & \operatorname{div}\left(|\nabla v|^{p-2} \nabla v\right) \\
= & -\gamma^{p}(p-1)\left(\psi^{\prime}\right)^{p-2} \psi^{\prime \prime}\left|\nabla W_{b}\right|^{p} \\
& -\left(\psi^{\prime}\right)^{p-1} \gamma^{p-1} \operatorname{div}\left(\left|\nabla W_{b}\right|^{p-2} \nabla W_{b}\right) \\
= & -\gamma^{p}(p-1)\left(h(\psi(t))^{p-1} h^{\prime}(\psi(t))\left|\nabla W_{b}\right|^{p}+\gamma^{p-1} b h^{p-1}(v) \geq\right. \\
& \gamma^{p-1} b h^{p-1}(v)
\end{aligned}
$$

$$
\geq\left(\frac{\gamma}{v}\right)^{p-1} b v^{p-1} \hat{g}^{p-1}(v) \geq b g^{p-1}(v) .
$$

We have used (11) in the last inequality. Since $\psi(0)=0$, it is clear that $v(x) \rightarrow 0$ as $|x| \rightarrow \infty$.

Since $\gamma \geq 1$, observe that the solution $v$ constructed in Lemma 3 also satisfies

$$
\begin{aligned}
& -\operatorname{div}\left(|\nabla v|^{p-2} \nabla v\right) \geq b \hat{g}^{p-1}(v) . \\
& \left(\widehat{G}_{1}\right) g \in C^{1}((0, \infty),(0, \infty)) ; \\
& \left(\widehat{G}_{2}\right) \lim _{u \rightarrow 0} \frac{g(u)}{u^{p-1}}=+\infty ; \\
& \left(\widehat{G}_{3}\right) \lim _{u \rightarrow \infty} \frac{g(u)}{u^{p-1}}=0 .
\end{aligned}
$$

Lemma 4. Assume that $g$ satisfies $\left[\widehat{G}_{1}\right]-\left[\widehat{G}_{3}\right]$. Then there exist functions $\underline{g}_{1}, \bar{g}_{1}$ such that

(i) $\underline{g}_{1}, \bar{g}_{1} \in C^{1}((0, \infty),(0, \infty))$;

(ii) $\underline{g}_{1}(s) \leq \frac{g(s)}{(s+1)^{p-1}} \leq \bar{g}_{1}(s), \forall s>0$ and

$$
\lim _{s \rightarrow 0^{+}} \underline{g}_{1}(s)=\lim _{s \rightarrow 0^{+}} \bar{g}_{1}(s)=\infty ;
$$

(iii) $g_{1}, \bar{g}_{1}$ are non-increasing on $(0, \infty)$;

(iv) $\varlimsup_{\lim _{s \rightarrow \infty}} g_{1}(s)=\lim _{s \rightarrow \infty} \bar{g}_{1}(s)=0$.

Proof.

$$
\begin{aligned}
& \lim _{s \rightarrow 0} \frac{g(s)}{(s+1)^{p-1}}=\lim _{s \rightarrow 0} \frac{g(s)}{(s)^{p-1}}\left(\frac{s}{s+1}\right)^{p-1}=+\infty, \\
& \lim _{s \rightarrow \infty} \frac{g(s)}{(s+1)^{p-1}}=\lim _{s \rightarrow \infty} \frac{g(s)}{(s)^{p-1}}\left(\frac{s}{s+1}\right)^{p-1}=0 .
\end{aligned}
$$

We set $\bar{g}(s)=\sup _{t \geq s>0} \frac{g(t)}{(t+1)^{p-1}}$, then we have

$\bar{g}(s) \geq \frac{g(t)}{(t+1)^{p-1}}, \forall s>0$ and $t \geq s$

and $\bar{g}(s)$ is non-increasing on $(0, \infty)$. Moreover,

$$
\lim _{s \rightarrow 0^{+}} \bar{g}(s)=\infty \text { and } \lim _{s \rightarrow \infty} \bar{g}(s)=0 .
$$

Now we can assume $\bar{g} \in C^{1}(0, \infty)$. If not, we can replace it by

$$
\bar{g}_{1}(s)=\frac{2}{s} \int_{\frac{s}{2}}^{s} \bar{g}(t) d t, s>0
$$

Obviously,

$$
\bar{g}(s) \leq \bar{g}_{1}(s) \leq \bar{g}\left(\frac{s}{2}\right), \forall s>0 ;
$$

and 


$$
\begin{gathered}
\bar{g}_{1}^{\prime}(s)=\frac{2}{s}\left(\bar{g}(s)-\frac{1}{2} \bar{g}\left(\frac{s}{2}\right)\right)-\frac{2}{s^{2}} \int_{\frac{s}{2}}^{s} \bar{g}(t) d t \\
\leq \frac{2}{s}\left(\bar{g}(s)-\frac{1}{2} \bar{g}\left(\frac{s}{2}\right)\right)-\frac{2}{s^{2}} \frac{s}{2} \bar{g}(s)=\frac{1}{s}\left(\bar{g}(s)-\bar{g}\left(\frac{s}{2}\right)\right) \leq 0 . \\
\text { i.e., } \bar{g}_{1} \in C^{1}((0, \infty),(0, \infty)) . \\
\underline{g}(s)=\inf _{s \geq t>0} \frac{g(t)}{(t+1)^{p-1}}
\end{gathered}
$$

Observe that

$$
0<\underline{g}(s) \leq \frac{g(t)}{(t+1)^{p-1}}, \quad \forall s>0 ;
$$

and $\underline{g}(s)$ is non-increasing on $(0, \infty)$. Moreover,

$\lim _{s \rightarrow 0^{+}} \underline{g}(s)=\infty$ and $\lim _{s \rightarrow \infty} g(s)=0$.

Now we can assume $g \in C^{1}(0, \infty)$. If not, we can replace it by

$$
\underline{g}_{1}(s)=\frac{2}{s} \int_{s}^{s+1} \underline{g}(t) d t, s>0
$$

Obviously,

$$
\underline{g}(s+1) \leq \underline{g}_{1}(s) \leq \underline{g}(s), \forall s>0
$$

and

$$
\begin{gathered}
\underline{g}_{1}^{\prime}(s)=\underline{g}(s+1)-\underline{g}(s) \leq 0, \forall s>0 \\
\text { i.e., } \underline{g}_{1} \in C^{1}((0, \infty),(0, \infty)) .
\end{gathered}
$$

\section{Proof of Main Theorems}

In this section, we prove our main results.

Theorem 1. Let $\Omega \subseteq \mathbf{R}^{N}$ be a bounded smooth domain that contains $B(0,1)$, the ball of radius one centered at the origin, and let $f$ satisfies $\left[F_{1}\right],\left[F_{2}\right]$ and $\left[F_{3}\right]$, where $b$ satisfies $\left[B_{1}\right],\left[B_{3}\right]$ and $g$ satisfies $\left[G_{1}\right],\left[G_{2}\right]$. Then the problem

$$
\left\{\begin{array}{l}
-\operatorname{div}\left(|\nabla u|^{p-2} \nabla u\right)=f(x, u), x \in \Omega \\
u(x)=0, x \in \partial \Omega
\end{array}\right.
$$

has a positive solution $u$ in $C^{1, \alpha}(\Omega) \bigcap C(\bar{\Omega})$ such that $u \geq \phi_{\Omega}$ where $\varphi_{\Omega}$ is an eigenfunction of the eigenvalue problem (4) on $\Omega$ with $c(x)=a(x)$ normalized such that $0<\phi_{\Omega} \leq \vartheta$ on $\Omega$. Here $\vartheta$ is the constant in condition $\left[F_{3}\right]$.

Proof. Let $W_{\Omega}$ be the solution of (5) and set $v_{\Omega}(x)=\eta^{-1}\left(\gamma W_{\Omega}\right)$ where $\eta$ and $\gamma$ are defined as in (9) and (10) respectively. Then $v_{\Omega}=0$ on $\partial \Omega$, and proceeding as in the proof of Lemma 3, we note that

$$
-\operatorname{div}\left(\left|\nabla v_{\Omega}\right|^{p-2} \nabla v_{\Omega}\right) \geq b(x) g^{p-1}\left(v_{\Omega}\right), x \in \Omega .
$$

Therefore, by condition $\left[F_{2}\right]$, we see that

$$
-\operatorname{div}\left(\left|\nabla v_{\Omega}\right|^{p-2} \nabla v_{\Omega}\right) \geq f\left(x, v_{\Omega}\right), x \in \Omega .
$$

We recall, by the above, that $v$ also satisfies

$$
-\operatorname{div}\left(\left|\nabla v_{\Omega}\right|^{p-2} \nabla v_{\Omega}\right) \geq b(x) \hat{g}^{p-1}\left(v_{\Omega}\right), x \in \Omega .
$$

Let $\Omega$ be a smooth bounded domain that contains $B(0,1)$ the unit ball centered at the origin. Now, let $\phi_{\Omega}$ be the first eigenfunction of the problem (4) with $c(x)=a(x)$ such that $0<\phi_{\Omega} \leq \vartheta$, where $\vartheta$ is the positive constant in $\left[F_{3}\right]$. Invoking conditions $\left[F_{2}\right]$ and $\left[F_{3}\right]$, we get

$$
\begin{aligned}
& -\operatorname{div}\left(\left|\nabla \phi_{\Omega}\right|^{p-2} \nabla \phi_{\Omega}\right) \\
& =\lambda_{\Omega} a(x) \phi_{\Omega}^{p-1} \leq \lambda_{1} a(x) \phi_{\Omega}^{p-1} \leq f\left(x, \phi_{\Omega}\right), \quad x \in \Omega
\end{aligned}
$$

Moreover, since $0<\varphi_{\Omega} \leq 1$, we also note that,

$$
\begin{aligned}
& -\operatorname{div}\left(\left|\nabla \phi_{\Omega}\right|^{p-2} \nabla \phi_{\Omega}\right) \leq f\left(x, \phi_{\Omega}\right) \\
& \quad \leq b(x) g^{p-1}\left(\phi_{\Omega}\right) \leq b(x) \hat{g}^{p-1}\left(\phi_{\Omega}\right), \quad x \in \Omega
\end{aligned}
$$

Therefore, we get

$$
-\operatorname{div}\left(\left|\nabla \phi_{\Omega}\right|^{p-2} \nabla \phi_{\Omega}\right) \leq b(x) \hat{g}^{p-1}\left(\phi_{\Omega}\right), \quad x \in \Omega
$$

Recalling that $\hat{g}$ is non-increasing, by Lemma 1 we note, from (15) and (17), that $\phi_{\Omega} \leq v_{\Omega}$. Then by the elliptic regularity theory and Lemma 2, (14) and (16), we conclude that (12) has a solution $u$ such that $\phi_{\Omega} \leq u \leq v_{\Omega}$ and $u \in C^{1, \alpha}(\Omega) \bigcap C(\bar{\Omega})$.

Let $W_{\Omega}$ be as in the proof of the above Theorem. Then we note that $W_{\Omega} \leq W_{b}$, and hence $v_{\Omega} \leq v$ where $v$ is as in Lemma 3. Then we deduce a non-singular case.

Theorem 2. If $\mathrm{f}$ satisfies $\left[F_{1}\right], f(x, 0)=0$, where $b$ satisfies $\left[B_{1}\right],\left[B_{3}\right]$ and $g$ satisfies $\left[G_{1}\right],\left[G_{2}\right]$, then problem (1) has a solution $u \in C_{l o c}^{1, \alpha}\left(\mathbf{R}^{N}\right)$.

Proof. For each positive integer $k$, let $B_{k}=B(0, k)$ be the ball of radius $k$ centered at the origin. By Theorem 1, for each positive integer $k$ we let $u_{k} \in C^{1, \alpha}\left(B_{k}\right)$ be a solution of

$$
\left\{\begin{array}{l}
-\operatorname{div}\left(|\nabla u|^{p-2} \nabla u\right)=f(x, u), x \in B_{k} \\
u(x)=0, \quad x \in \partial B_{k}
\end{array}\right.
$$

Then $0 \leq u_{k}(x) \leq v_{k}(x)$ in $B_{k}$, where $v_{k}$ are as in Theorem 1. Corresponding to the ball $B_{k}$. It is easy to see that 0 is a subsolution.

Recall the above, $v \geq v_{k}$ on $B_{k_{-}}$for all $k \geq 1$, and hence $0 \leq u_{k}(x) \leq v(x)$ for all $x \in \bar{B}_{2}$.

By a standard procedure, we conclude that $\left\{u_{k}\right\}_{l}^{\infty}$ has a subsequence that converges uniformly to a function in $C^{2, \alpha}(\Omega)$. By a diagonalization process it follows that $\left\{u_{k}\right\}$ has a subsequence that converges uniformly on open bounded subsets of $\mathbf{R}^{N}$ to $u \in C_{l o c}^{2, \alpha}\left(\mathbf{R}^{N}\right)$ and that $u$ is a solution of (1). Since $0 \leq u \leq v$, it follows 
that $u(x) \rightarrow 0$ as $|x| \rightarrow \infty$.

In the last part of the paper, we prove a nonexistence result for the following problem,

$$
\left\{\begin{array}{l}
-\operatorname{div}\left(|\nabla u|^{p-2} \nabla u\right) \\
\quad=d(x)\left[g(u)+r(u)+|\nabla u|^{q}\right], u>0, \text { in } \mathbf{R}^{N} \\
u(x) \rightarrow 0, \text { as }|x| \rightarrow \infty
\end{array}\right.
$$

The result solves an open problem in [4] for $p>1$, $q \geq 0$ for the case when $u$ is a radially symmetric solution. Before the proof, we state some conditions which we needed at the below.

$$
\begin{gathered}
\left(\widehat{G}_{1}\right) g \in C^{1}((0, \infty),(0, \infty)) ; \\
\left(\widehat{G}_{2}\right) \lim _{u \rightarrow 0} \frac{g(u)}{u^{p-1}}=+\infty ; \\
\left(\widehat{G}_{3}\right) \lim _{u \rightarrow \infty} \frac{g(u)}{u^{p-1}}=0 .
\end{gathered}
$$

Theorem 3. Suppose $\left(\widehat{G}_{1}\right)-\left(\widehat{G}_{3}\right)$ are fulfilled and $d$ is a positive radial function, $r$ is a nonnegative radial function that is continuous on $\mathbf{R}^{N}$ and satisfies

$$
\int_{0}^{\infty}\left(\xi^{1-N} \int_{0}^{\xi} d(\sigma) \sigma^{N-1} d \sigma\right)^{\frac{1}{p-1}} d \xi=\infty
$$

then the problem (19) has no positive radial solution that decays to zero near infinity.

Proof. Suppose (19) has such a solution $u(r)$. Then

$$
\begin{aligned}
& -\operatorname{div}\left(|\nabla u(r)|^{p-2} \nabla u(r)\right) \\
& =d(r)\left[g(u(r))+r(u(r))+|\nabla u(r)|^{q}\right],
\end{aligned}
$$

or, equivalently, $u(r)$ is a solution to the problem

$$
-\left(r^{N-1}\left|u^{\prime}\right|^{p-2} u^{\prime}\right)^{\prime} \geq r^{N-1} d(r)[g(u(r))+r(u(r))]
$$

Integrating (20) from 0 to $r$, we have

$$
-r^{N-1}\left|u^{\prime}\right|^{p-2} u^{\prime} \geq \int_{0}^{r} \sigma^{N-1} d(\sigma)[g(u(\sigma))+r(u(\sigma))] d \sigma .
$$

Hence $u^{\prime}(r)<0$; i.e., $u(r)$ is non-increasing. We put $\ln (u(r)+1)=\tilde{u}(r)>0$ for all $r>0$. Then we have

$$
\begin{aligned}
& \operatorname{div}\left(|\nabla \tilde{u}(r)|^{p-2} \nabla \tilde{u}(r)\right) \\
& =\left(\frac{1}{u+1}\right)^{p-1} \operatorname{div}\left(|\nabla u(r)|^{p-2} \nabla u(r)\right)-(p-1) \frac{1}{(u+1)^{p}}|\nabla u|^{p},
\end{aligned}
$$
and $\tilde{u}(r)$ satisfies

$$
\begin{aligned}
& \left(\left|\tilde{u}^{\prime}\right|^{p-2} \tilde{u}^{\prime}\right)^{\prime}+\frac{N-1}{r}\left|\tilde{u}^{\prime}\right|^{p-2} \tilde{u}^{\prime} \\
& +(p-1) \frac{1}{(u+1)^{p}}|\nabla u|^{p} \leq-d(r) \frac{g(u(r))+r(u(r))}{(u+1)^{p-1}}
\end{aligned}
$$

Multiplying Equation (21) by $r^{N-1}$ and integrating on $(0, \xi)$ yields

$$
\begin{aligned}
& \xi^{N-1}\left|\tilde{u}^{\prime}\right|^{p-2} \tilde{u}^{\prime}+\int_{0}^{\xi} \frac{(p-1) \sigma^{N-1}}{(u+1)^{p}}|\nabla u|^{p} d \sigma \\
& \leq-\int_{0}^{\xi} \sigma^{N-1} d(\sigma) \frac{g(u(\sigma))+r(u(\sigma))}{(u+1)^{p-1}} d \sigma
\end{aligned}
$$

If $p$ is even, we can deduce that

$$
\begin{aligned}
& \tilde{u}(r)-\tilde{u}(0)+\int_{0}^{r}\left(\xi^{1-N} \int_{0}^{\xi} \frac{(p-1) \sigma^{N-1}}{(u+1)^{p}}|\nabla u|^{p} d \sigma\right)^{\frac{1}{p-1}} d \xi \\
& \leq-\int_{0}^{r}\left(\xi^{1-N} \int_{0}^{\xi} \sigma^{N-1} d(\sigma) \frac{g(u(\sigma))+r(u(\sigma))}{(u+1)^{p-1}} d \sigma\right)^{\frac{1}{p-1}} d \xi
\end{aligned}
$$

We observe that $u(r)<u(0)$ for all $r>0$ and $\tilde{u}(r)<\tilde{u}(0)$ for all $r>0$.

Since $\tilde{u}(r)$ is positive, then (23) implies

$$
\int_{0}^{r}\left(\xi^{1-N} \int_{0}^{\xi} \sigma^{N-1} d(\sigma) \frac{g(u(\sigma))+r(u(\sigma))}{(u+1)^{p-1}} d \sigma\right)^{\frac{1}{p-1}} d \xi \leq \tilde{u}(0)
$$

for all $r>0$. Now, using Lemma 4 in (24), we have

$$
\begin{gathered}
\int_{0}^{r}\left(\xi^{1-N} \int_{0}^{\xi} \sigma^{N-1} d(\sigma) \underline{g}_{1}(u(\sigma)) d \sigma\right)^{\frac{1}{p-1}} d \xi \\
\leq \int_{0}^{r}\left(\xi^{1-N} \int_{0}^{\xi} \sigma^{N-1} d(\sigma) \frac{g(u(\sigma))}{(u(\sigma)+1)^{p-1}} d \sigma\right)^{\frac{1}{p-1}} d \xi \\
\leq \int_{0}^{r}\left(\xi^{1-N} \int_{0}^{\xi} \sigma^{N-1} d(\sigma) \frac{g(u(\sigma))+r(u(\sigma))}{(u(\sigma)+1)^{p-1}} d \sigma\right)^{\frac{1}{p-1}} d \xi \leq \tilde{u}(0) .
\end{gathered}
$$

If $p$ is odd, we deduce that

$$
\begin{gathered}
\tilde{u}(r)-\tilde{u}(0) \geq \int_{0}^{r}\left(\xi^{1-N} \int_{0}^{\xi} \frac{(p-1) \sigma^{N-1}}{(u+1)^{p}}|\nabla u|^{p} d \sigma\right)^{\frac{1}{p-1}} d \xi \\
\quad+\int_{0}^{r}\left(\xi^{1-N} \int_{0}^{\xi} \sigma^{N-1} d(\sigma) \frac{g(u(\sigma))+r(u(\sigma))}{(u+1)^{p-1}} d \sigma\right)^{\frac{1}{p-1}} d \xi
\end{gathered}
$$

then (25) implies

$$
\begin{aligned}
& \int_{0}^{r}\left(\xi^{1-N} \int_{0}^{\xi} \sigma^{N-1} d(\sigma) \frac{g(u(\sigma))+r(u(\sigma))}{(u+1)^{p-1}} d \sigma\right)^{\frac{1}{p-1}} d \xi \\
& \leq \tilde{u}(r)<\tilde{u}(0)
\end{aligned}
$$

for all $r>0$. As the same as the above we get

$$
\int_{0}^{r}\left(\xi^{1-N} \int_{0}^{\xi} \sigma^{N-1} d(\sigma) \underline{g}_{1}(u(\sigma)) d \sigma\right)^{\frac{1}{p-1}} d \xi<\tilde{u}(0) .
$$

But, since $\underline{g}_{1}$ is non-increasing on $(0, \infty)$, we have 


$$
\infty=\int_{0}^{r}\left(\xi^{1-N} \int_{0}^{\xi} \sigma^{N-1} d(\sigma) d \sigma\right)^{\frac{1}{p-1}} d \xi<\frac{\tilde{u}(0)}{\underline{g}_{1}^{\frac{1}{p-1}}(u(0))}<\infty,
$$

which is a contradiction.

\section{References}

[1] G. Astrita and G. Marrucci, "Principles of Non-Newtonian Fluid Mechanics," McGraw-Hill, Rochester, 1974.

[2] L. K. Martinson and K. B. Pavlov, "Unsteady Shear Flows of a Conducting Fluid with a Rheological Power Law, Magnit," Gidrodinamika, Vol. 2, 1971, pp. 50-58.

[3] J. R. Esteban and J. L. Vazquez, "On the Equation of Turbulent Filtration in One-Dimensional Porous Media," Nonlinear Analysis, Vol. 10, No. 11, 1982, pp. 13031325.

[4] Z. M. Guo, "Existence and Uniqueness of the Positive Radial Solutions for a Class of Quasilinear Elliptic Equations," Applied Analysis, Vol. 47, No. 3, 1992, pp. 173-190.

[5] Z. M. Guo, "Some Existence and Multiplicity Results for a Class of Quasilinear Elliptic Equatons," Nonlinear Analysis, Vol. 18, No. 10, 1992, pp. 957-971.

[6] Z. M. Guo and J. R. L. Webb, "Uniqueness of Positive Solutions for Quasilinear Elliptic Equations When a Parameter is Large," Proceedings of Royal Society of Edinburgh, Edinburgh, Vol. 124, No. 1, 1994, pp. 189198.

[7] M. G. Crandall, P. H. Rabinowitz and L. Tartar, "On a Dirichlet Problem with a Singular Nonlinearity," Cотmunications in Partial Differential Equations, Vol. 2, No. 2, 1977, pp. 193-222.

[8] S. Cui, "Existence and Nonexistence of Positive Solutions for Singular Semilinear Elliptic Boundary Value Problems," Nonlinear Analysis, Vol. 41, No. 1-2, 2000, pp. 149-176.

[9] A. V. Lair and A. W. Shaker, "Classical and Weak Solutions of a Singular Semilinear Elliptic Problem," Journal of Mathematical Analysis and Applications, Vol.
211, No. 2, 1997, pp. 371-385.

[10] A. C. Lazer and P. J. McKenna, "On a Singular Nonlinear Elliptic Boundary-Value Problem," Proceedings of the American Mathematical Society, Vol. 111, No. 3, 1991, pp. 721-730.

[11] H. Maagli and M. Zribi, "Existence and Estimates of Solutions for Singular Nonlinearity Elliptic Problems," Journal of Mathematical Analysis and Applications, Vol. 263, No. 2, 2001, pp. 522-542.

[12] J. Shi and M. Yao, "Positive Solutions for Elliptic Equations with Singular Nonlinearity," Electronic Journal of Qualitative Theory of Differential Equations, Vol. 4, 2005, pp. 1-11.

[13] H. Xue and Z. Zhang, "A Remark on Ground State Solutions for Lane-Emden-Fowler Equations with a Convection Term," Electronic Journal of Differential Equations, Vol. 2007, No. 53, 2007, pp. 1-10.

[14] H. Brezis and S. Kamin, "Sublinear Elliptic Equations in $R^{N}$, Manuscripta Mathematica, Vol. 74, No. 1, 1992, pp. 87-106.

[15] S. Wu and H. Yang, "The Existence Theorems for a Class of Sublinear Elliptic Equations in $R^{N}, "$ Acta Mathematica Sinica, Vol. 13, No. 3, 1997, pp. 259-304.

[16] Z. Zhang, "A Remark on the Existence of Entire Solutions of a Singular Semilinear Elliptic Problem," Journal of Mathematical Analysis and Applications, Vol. 215, No. 2, 1997, pp. 579-582.

[17] Z. Zhang, "A Remark on the Existence of Positive Entire Solutions of a Sublinear Elliptic Problem," Nonlinear Analysis, Vol. 67, 2007, pp. 147-153.

[18] K. E. Mabrouk, "Entire Bounded Solutions for a Class of Sublinear Elliptic Equations," Nonlinear Analysis, Vol. 58, No. 1-2, 2004, pp. 205-218.

[19] J. V. Goncalves and C. A. Santos, "Existence and Asymptotic Behavior of Non-Radially Symmetric Ground States of Semilinear Singular Elliptic Equations," Nonlinear Analysis, Vol. 65, No. 4, 2006, pp. 719-727.

[20] A. Mohammed, "Ground State Solutions for Singular Semilinear Elliptic Equations," Nonlinear Analysis, Vol. 71, No. 3-4, 2009, pp. 1276-1280. 